\title{
Work environment and neck and shoulder pain: the influence of exposure time. Results from a population based case-control study
}

\author{
K Fredriksson, L Alfredsson, G Ahlberg, M Josephson, Å Kilbom, E Wigaeus Hielm, \\ C Wiktorin, E Vingård, the MUSIC/Norrtälje Study Group
}

See end of article for authors' affiliations

Correspondence to: Dr K Fredriksson, Statistics Sweden, Box 24300 SE-104 51 Stockholm, Sweden;

kerstin.fredriksson2@scb.se

Accepted 18 September 2001

\begin{abstract}
Objectives: To study associations between long term and short term exposure to different work environmental conditions and the incidence of neck or shoulder pain. The results were obtained as part of the MUSIC-Norrtälje study, which is a population based case-control study conducted in Sweden in 1993-7.

Methods: The cases were people from the study base who sought medical care or treatment for neck or shoulder pain. Information on physical and psychosocial conditions in the work environment, currently and 5 years ago, and lifestyle factors, was obtained by self administered questionnaires from 310 cases and 1277 randomly selected referents.

Results: Associations between both physical and psychosocial exposures in the work environment and seeking care for neck or shoulder pain were found. The risk patterns differed for the sexes, and risk ratios exceeding 1.5 were more often found among women than among men. Generally, subjects who had experienced a recent increase of exposure were more likely (relative risk (RR) 2.1-3.7) to seek care than those who had been exposed long term (RR 1.5-1.8). Among women, an increased amount of visual display terminal (VDT) work, work above shoulder level, and reduced opportunities to acquire new knowledge, and among men, an increased amount of seated work were associated with neck or shoulder pain. This might indicate short induction periods for neck or shoulder pain for these exposures. However, for repetitive work with the hands and hindrance at work among women, and possibly also local vibrations among men, the induction periods seem to be longer. Interactive effects between factors, both at work and in the family, were found, but only among women.

Conclusions: Associations between some exposures in the work environment and seeking care for neck or shoulder pain were found. The high RRs for short term exposure might indicate that for many factors the induction period for neck or shoulder pain is short.
\end{abstract}

$\mathrm{N}$ eck and shoulder disorders are prevalent among both women and men. Work related factors, both of physical and psychosocial origin, as well as lifestyle factors, have been identified as being associated with a high prevalence and incidence of neck or shoulder disorders. Repetitive hand and finger movements and monotonous work tasks, ${ }^{1-9}$ awkward postures, ${ }^{2}{ }^{10}{ }^{11}$ duration of sitting and twisting and bending of the trunk, ${ }^{12}$ adverse psychosocial conditions at work, ${ }^{6}{ }^{13} 14$ and smoking ${ }^{415}$ are examples of such factors.

Earlier studies on neck or shoulder pain have mostly been made among workers who are highly exposed to specific factors at work; however, risk factors identified from such studies are highly unusual in most jobs. Nevertheless, neck or shoulder pain is very common in the general population. The rapidly changing conditions in working life today, and the increase in mobility of workers also present new challenges to researchers. $^{17}$

Our intention in this study is to deepen the knowledge about which factors influence the incidence of neck or shoulder pain in the general population, and also to study questions related to time of exposure. Are there any differences where neck and shoulder pain are concerned, between having adverse physical and psychosocial conditions over a long period and experiencing the same adverse conditions only during the most recent year? The aim of the present study was to study the impact of (a) prevailing high exposure to various work related factors during a 5 year period on the incidence of neck or shoulder pain, and (b) a shift from an exposure at a low level to the same exposure at a high level during the same 5 year period.

\section{MATERIALS AND METHODS \\ Study population}

The present study is a part of the Musculoskeletal Intervention Centre (MUSIC)- Norrtälje study, which is a population based case-control study of low back and neck or shoulder pain. ${ }^{18-20}$ The study population comprised all men and women, 20-59 years of age, living in the district of Norrtälje and not working or studying outside this area, about 17000 people in all. Norrtälje is in the centre of Sweden about $50 \mathrm{~km}$ north of Stockholm. The study period for this part of the study was 3 years, from 15 June 1994 to 15 June 1997.

\section{Cases}

The cases were people from the study base who during the study period sought medical care or treatment for neck or shoulder pain from any of the 78 caregivers (4l physicians, 23 physiotherapists, seven chiropractors and Drs of naprapathy, seven osteopaths, homeopaths, etc) who were active in the area. The aim was to study incident cases, and therefore all caregivers were asked to send new cases, defined as patients

Abbreviations: MUSIC, musculoskeletal intervention centre; RPE, ratings of perceived exertion; RR, relative risk; VDT, visual display terminal 
not having sought care or been treated for low back or neck or shoulder pain during the previous 6 months. No caregiver refused to participate. According to interviews with the caregivers, only a few patients refused to participate, but there are no figures available to back up this information.

\section{Controls}

Controls were selected from the study base as a stratified random sample by means of the population register, taking age (in 5 year intervals) and sex into consideration (category matching). At least one control was selected per case. If there was space in the investigation schedule a new control within the same 5 year span as the last case was randomly selected and invited to the study. Controls who were found to have sought medical care for low back or neck or shoulder pain within the past 6 months were excluded. Altogether 2500 controls were enrolled and the participation rate was $69 \%$.

For this particular study the study base was restricted to people with a job both during the year preceding the examination and 5 years earlier (69 cases and 431 controls were excluded for this reason). Further, people reporting changes of occupation due to neck or shoulder disorders were excluded ( 13 cases and 17 controls). Altogether 210 female cases and 698 female controls, and 100 male cases and 579 male controls, were included in the analyses.

An additional 224 subjects, who also fulfilled the inclusion criteria, only answered the questionnaires but did not attend the interview or the examination. Data from these subjects were used for additional analyses.

\section{Data collection}

Cases and controls filled in questionnaires about background data, earlier musculoskeletal disorders, pain and function, and current as well as earlier physical conditions at work.

The participants were also examined by six experienced physiotherapists (blinded for the case or control status of the participants) and classified into one of the following four groups on the basis of signs shown during the examination: (a) without any objective signs of tension neck syndrome, shoulder tendinitis, or cervical rhizopathy, $(b)$ tension neck syndrome without any signs of shoulder involvement, (c) shoulder tendinitis with or without signs of neck involvement, (d) cervical rhizopathy. The interrater reliability was found to be good to excellent for examination of the low back, ${ }^{21}$ and there is no reason to think that the reliability is lower for examination of the neck and shoulders.

Information about current and earlier psychosocial conditions at work and organisational conditions was collected by interviews, which were conducted by seven well trained interviewers, blinded for the case or control status of the participants, and using regular meetings to remain constant in assessment over time and in relation to each other. ${ }^{22}$

The questionnaire on physical loads included questions on conditions presumed to be relevant for neck or shoulder pain. ${ }^{18}{ }^{20}$ Exposure information was collected about two points in time, current exposure and exposure 5 years back in time. An ordinal five point scale, for number of exposed days per week or month, was used for most of the questions. For questions about percentage of time spent working in a seated position, working at a visual display terminal (VDT), working with hand held vibrating tools, or with domestic work, a continuous visual analogue scale was used. For one question about the level of general perceived workload a semicontinuous scale was used. ${ }^{23}$ Frequency of bending and twisting or hand and finger movements was described as movements performed in the same fashion many times each hour or each minute, respectively.

For all physical exposure factors, cut off points, classifying the subjects into one exposed and one unexposed group, were based on the lowest level of exposure the authors thought would be harmful for neck or shoulder pain. The cut off point for VDT work was chosen from results derived from earlier studies. ${ }^{24}$ the cut off points for time spent sitting and perceived general workload were based on the distribution among controls and chosen so that about $25 \%$ of the most highly exposed controls would be considered to be exposed. The cut off points are shown in table 1.

For psychosocial loads at work, the participants were asked about their degree of participation in work planning, opportunities to acquire new knowledge and to use existent knowledge, degree of support from colleagues and superiors, and potential hindrance at work. The criteria for assessing if hindrance existed were risks of obvious loss of quality of the performed work, risks that a delay would result in extra work, or an apparent risk of accidents or illness. ${ }^{22}{ }^{25}$ The exposure information was dichotomised, and for participation in work planning the workers who had any influence were compared with those for whom there was a fixed schedule or the employer decided everything about the work. Subjects who answered that they could use their professional knowledge at work and needed to acquire new knowledge to be able to perform work, to some extent or mostly, were compared with those who answered not at all or to a limited extent. Subjects who considered that either colleagues or superiors (or both) mostly or always gave support were compared with those who answered never or mostly not. Hindrance with tasks or goals, material resources, personal resources, resources for knowledge, environmental conditions, customers, physical conditions, and society, were assessed on a scale $1-4$ for each item separately. The degrees of hindrance at work were summed up, and if the sum showed a high degree of hindrance for at least one of the items included, or more than irritating hindrance for at least more than half of the included items, the subject was considered to be hindered. The participants were asked about current conditions and retrospectively, if the situation had been the same or if there had been any changes, for the worse or for the better, within the preceding 5 year period. $^{26}$

Smoking (dichotomised into smoking regularly $v$ no smoking during the previous 5 year period), sport activities (no or little physical activity during leisure time $v$ at least 20 minutes of sport activities regularly/week), overtime work ( $\leqslant 40$ hours work/week $v>40$ hours work/week) and irregular working hours or shift work (irregular working hours or shift work $v$ ordinary daytime work) were assessed by a self administered questionnaire. These factors were considered as potential confounders together with age (in 5 year age groups), earlier symptoms of neck or shoulder disorders (duration $>3$ months on any single occasion), and socioeconomic status (four groups: blue collar workers, white collar workers in lower or intermediate positions, white collar workers in senior positions, and self employed people or employers).

\section{Data analysis}

All analyses were performed separately for women and men. Cases and controls were compared for their physical and psychosocial conditions in two separate analyses. (1) Subjects reporting a presumed harmful condition both currently and 5 years ago (long term exposed) were contrasted with subjects reporting no exposure at either of these points (the unexposed group). (2) Subjects reporting current exposure but no exposure at the earlier time (short term exposed, had increased their exposure during the 5 year period) were contrasted with the same unexposed group. For physical factors an increase of exposure was defined as adverse exposure now but not 5 years ago. For psychosocial factors the definition of increase of exposure was that of being exposed to harmful conditions currently but also reporting that conditions were better earlier within the 5 year period.

The relation between different exposures and seeking care for neck or shoulder pain was estimated by calculating the 


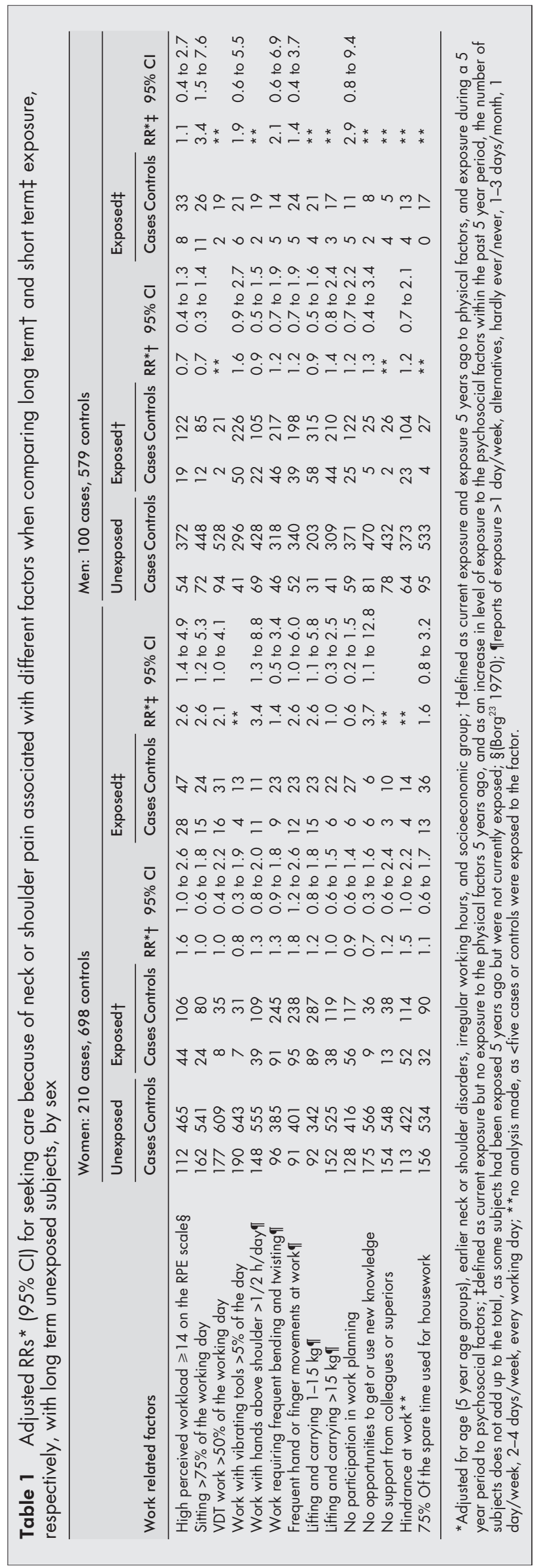

odds ratio with 95\% confidence intervals (95\% CIs) with PROC LOGISTIC in SAS statistical software. ${ }^{27}$ The odds ratio was interpreted as an estimate of the incidence rate ratio ( $R R$ ) because the design was that of a population based case-control study. ${ }^{28}$ The analyses were first carried out with adjustment for age ( 5 year age groups), lifestyle factors, earlier symptoms of neck or shoulder pain for more than 3 months, conditions affecting working time, and socioeconomic status. Factors with the lower $95 \%$ confidence limit $\geqslant 0.9$ in these analyses were then included in a multivariate analysis, to study all relevant factors simultaneously for incidence of neck or shoulder pain.

Potential excess risk due to interaction between physical and psychosocial factors at work, or between occupational and leisure time factors, for the risk of seeking care for neck or shoulder pain, was analysed by the method proposed by Rothman. ${ }^{29}$ To examine interaction between a factor A and a factor B we calculated the relative risk for subjects that reported both or either of them, contrasted with an unexposed group that reported neither factor. The proportion of excess risk due to interaction was calculated as:

$(\mathrm{RR}(\mathrm{A}+\mathrm{B})-\mathrm{RR}(\overline{\mathrm{A}}+\mathrm{B})-\mathrm{RR}(\mathrm{A}+\overline{\mathrm{B}})+\mathrm{l}) / \mathrm{RR}(\mathrm{A}+\mathrm{B}))$

with corresponding 95\% CI. Only interaction terms where the proportion of the excess risk was at least $20 \%$ and the RR for the interaction term was at least 2.0 with a lower confidence limit of 1.0 are reported.

A separate analysis was carried out for the 888 subjects in the study group who had answered in the questionnaire that they had never before experienced neck or shoulder pain for more than 7 consecutive days. If less than five cases or controls were exposed to a factor, the factor was excluded from the analysis.

\section{RESULTS}

The mean age was 43.4 years among women and 43.1 among men, ranging between 20 and 59 years of age. The age distribution formed an inverted $U$ shaped curve, somewhat displaced to the right among women. From the clinical examination (the median time between visiting a caregiver and examination was 2 weeks) $17 \%$ of the male cases and $20 \%$ of the female cases were classified as having tension neck syndrome. Of the male cases $31 \%$ and of the female cases $43 \%$ had signs of shoulder tendinitis with or without signs of neck involvement. About $10 \%$ of both female and male cases had signs of cervical rhizopathy. The remaining cases either had recovered from their pain or could not be classified into any special diagnosis, according to the criteria chosen. Among women, the percentage of cases and controls with a current blue collar job, was almost the same, although among men more cases than controls were blue collar workers (table 2).

\section{Long term exposure}

Regarding long term exposure, men reported more use of vibrating hand tools and more lifting and carrying than women. Women more often than men considered that they had to do most of the housework (table 1). Long term perceived high workload, long term exposure to frequent hand or finger work, and hindrance at work was associated with seeking care for neck or shoulder pain among women, whereas among men only a tendency for an association with long term exposure to work with vibrating tools was found (table 1). Adjustments for smoking, physical activity during leisure time, and overtime work only changed the RRs marginally, and were therefore not used in the analyses. 
Table 2 Cases (n (\%)) with neck or shoulder pain and controls among women and men in different socioeconomic groups

\begin{tabular}{|c|c|c|c|c|c|c|c|c|}
\hline \multirow[b]{2}{*}{ Socioeconomic group } & \multicolumn{4}{|c|}{ Women 210 cases, 698 controls } & \multicolumn{4}{|c|}{ Men 100 cases, 579 controls } \\
\hline & Cases & $\%$ & Controls & $\%$ & Cases & $\%$ & Controls & $\%$ \\
\hline Blue collar workers & 101 & 48 & 345 & 49 & 64 & 64 & 326 & 56 \\
\hline White collar workers in lower and intermediate positions & 84 & 40 & 241 & 35 & 20 & 20 & 138 & 24 \\
\hline White collar workers in senior positions & 14 & 6 & 81 & 12 & 9 & 9 & 65 & 11 \\
\hline Self employed people and employers & 7 & 3 & 23 & 3 & 7 & 7 & 39 & 7 \\
\hline
\end{tabular}

Table 3 Results from multivariate analysis among women for adjusted RRs* $(95 \% \mathrm{Cl})$ for seeking care because of neck or shoulder pain associated with different factors in subjects with long term exposure $\dagger$ compared with long term unexposed subjects (different analyses, one including factors with lower $95 \%$ confidence limit $\geqslant 0.9$ in table 2 and three separate analyses including interactive effects)

\begin{tabular}{|c|c|c|}
\hline \multirow[b]{2}{*}{ Multivariate analyses } & \multicolumn{2}{|c|}{ Women: 210 cases, 698 controls } \\
\hline & $\mathrm{RR}^{*} \dagger$ & $95 \% \mathrm{Cl}$ \\
\hline \multicolumn{3}{|l|}{ Work related factors: } \\
\hline High perceived workload $\geqslant 14$ on the RPE scale $\ddagger$ & 1.3 & 0.7 to 2.3 \\
\hline Hindrance at work & 1.5 & 0.9 to 2.5 \\
\hline Frequent hand or finger movements at work $\S$ & 1.9 & 1.2 to 3.0 \\
\hline \multicolumn{3}{|l|}{ Interactive effects between work related factors: } \\
\hline High perceived workload $\geqslant 14$ on the RPE scale $\neq$ +hindrance at work & 2.0 & 1.0 to 4.0 \\
\hline Hindrance at work but no high perceived workload & 1.0 & 0.6 to 1.7 \\
\hline High perceived workload but no hindrance at work & 0.9 & 0.4 to 1.6 \\
\hline \multicolumn{3}{|l|}{ Interactive effects between work related factors: } \\
\hline Work requiring frequent bending and twisting $\S+$ hindrance at work & 2.2 & 1.3 to 6.7 \\
\hline Work requiring frequent bending and twisting but no hindrance at work & 1.0 & 0.6 to 1.5 \\
\hline Hindrance at work but no work requiring frequent bending and twisting & 0.8 & 0.4 to 1.4 \\
\hline \multicolumn{3}{|l|}{ Interactive effects between work and non-work related factors: } \\
\hline Frequent hand or finger movements at work $\S+75 \%$ of the spare time used for housework & 2.3 & 1.2 to 4.3 \\
\hline Frequent hand or finger movements at work but not a lot of housework & 1.3 & 0.9 to 1.9 \\
\hline A lot of house work but not frequent hand or finger movements at work & 0.4 & 0.1 to 0.9 \\
\hline
\end{tabular}

However, adjustment for previous neck or shoulder disorders, socioeconomic status, and irregular working hours resulted in changes of some of the RRs, and these factors were therefore included. In a multivariate model for women, the estimated relative risks of seeking care were only marginally affected (table 3). Interactive effects on seeking care due to neck or shoulder pain were found among women and are shown in the second part of table 3. However, no interactive effects reaching the criteria for significance were found among men.

\section{Short term exposure}

For all items studied, except for VDT work, fewer subjects were exposed short term than long term within the 5 year period under study (table 1). In all socioeconomic groups there were subjects, both women and men, who had experienced changes towards less favourable conditions. Male blue collar workers more often reported adverse changes for more than one exposure factor than white collar workers did. Women who had experienced more than one change towards less favourable conditions were more evenly distributed among the socioeconomic groups.

Among both women and men, recent short term exposure resulted in substantially increased RRs compared with those derived from analysis of long term exposure (table 1). Due to the few exposed subjects, no interactive effects of short term exposure could be studied. When multivariate models were constructed for women, an increase in frequent hand and finger movements was no longer associated with seeking care for neck or shoulder pain. For the other factors all RRs were raised above the values displayed in table 1, especially for increased amount of VDT work, work above shoulder level, and fewer opportunities to acquire new knowledge, but the confidence intervals became wide and the results were unstable, so no final model could be constructed.

If the 30 subjects who were excluded from the analysis because they stated that they had changed jobs due to neck or shoulder disorders were re-added, only marginal changes in the RRs were found.

For the 224 subjects who did not attend the interview but did fill in the questionnaires, no separate analysis could be carried out, as these only included four female and three male cases. The proportion of these additional referents reporting various exposures was mostly similar to that of the study group. However, more women in this additional group reported perceived high workload, resulting in lowered RRs for both long term and short term exposure with 0.2 and 0.3 units respectively, when the additional subjects were added to the study group. Among men only small differences in the RRs were found when the additional subjects were added. These analyses were, however, not fully compatible with those performed for the original study group, as no adjustment for socioeconomic status could be made (no information on socioeconomic status was available for those subjects not attending the interview). No analyses could be made about psychosocial conditions, as all such data were gained from interview.

When data for the 888 subjects who had never before experienced neck or shoulder pain for more than 7 consecutive days were analysed, the difference between long term and short term exposure remained, although the $95 \%$ CIs became wider due to the few subjects. 
If the analyses were restricted to those subjects who received a confirmed diagnosis in the clinical examination, only minor changes in the relative risk estimates were found. The 95\% CIs became wider and there was a trend towards raised RR, especially for short term exposure.

\section{DISCUSSION}

In this population based case-control study, associations between seeking care for neck or shoulder pain and physical and psychosocial factors in the work environmental were found. There were no signs that a long exposure time increased the likelihood that a person would seek care-rather the opposite. Therefore it is suggested, based on this study, that the induction time for neck or shoulder pain due to work related conditions is relatively short, and that willingness to seek care is highest when the pain is a new experience. However, for some exposures, associations with seeking care were more consistent after 5 years of exposure.

The effects of cumulative physical workloads have been well established for arthrosis, ${ }^{30}{ }^{31}$ but few studies on other types of musculoskeletal disorders have been performed. The cumulative dose due to physical workload has usually been studied in terms of years of employment. An increase of disorders corresponding to an increase in the number of years at a particular job has been found among for example sewing machine operators, ${ }^{32}$ assembly workers, ${ }^{33}$ and medical secretaries ${ }^{34}$ pointing towards a dose-response effect of exposures in these jobs. It has also been found that the prevalence of neck disorders increased with the number of hours a day spent using VDTs, ${ }^{35}$ and percentage of time spent with shoulders abducted or raised. ${ }^{236}$

The mechanisms for cumulative trauma disorders in the neck or shoulder region are not well understood. Theories about an increased activity in the muscle spindle system, due to physical exposures, have been put forward. ${ }^{37}$ This increased activity could initiate a vicious circle, resulting in pain of the neck or shoulder muscles in work situations that involve repetitive work and awkward positions. Another theory is the so called "Cinderella syndrome theory", stating that the muscle fibres that are first used in a muscle contraction will also be the last to relax. ${ }^{38}$ This will lead to overuse of certain parts of the muscles and cause pain even if the loads are small. It has also been found that individual resting patterns are involved, and that the inability to relax the neck or shoulder muscles even if the muscles are doing no work predicts trapezius myalgia. ${ }^{39}$ However, none of these theories threw light on questions about duration of the induction period. The result in the present study suggests that the induction period is generally short.

\section{Risk factors related to work}

Short term rather than long term exposure to work above shoulder level, frequent bending and twisting at work and lifting loads, produced higher relative risks of neck or shoulder pain. Possible explanations of these differences are that people will adopt a better work technique or start to use lifting facilities or other ergonomic equipment after some time. The results for long term exposure may also be lowered by the so called healthy worker effect, ${ }^{40}$ - that is, only those who are very healthy stay in a demanding job. To partly account for such a selection bias, additional analysis of long term exposure was performed, where subjects who had experienced a decrease of exposure during recent years (those who were selected out of an occupation) were included among those with long term exposure. The relative risk estimates from those analyses only differed marginally compared with the analyses displayed in table 1 , and the difference between long and short term exposure was confirmed. However, there might have been selection out of an occupation earlier than during the observed 5 year period, which could not be accounted for.
Coping strategies and adaptation of soft tissues do not seem to be enough to prevent neck or shoulder pain leading to visits to caregivers after five years of exposure to repetitive work with the hands and fingers among women. This may also be the case for work with hand held vibrating tools among men.

There has been a great increase in VDT work during recent years, and this is also shown in our results. Associations between working at a VDT more than $50 \%$ of the working day and seeking care for neck or shoulder pain were only found among women exposed for less than 5 years. The explanation may be that people adopt better work techniques, or that reorganisation leads to a better distribution of work tasks after an introductory period. ${ }^{41}$

Sitting work for more than $95 \%$ of the working day has been identified as a risk factor for neck pain..$^{42}$ In the present study we could not do any analyses on sitting $95 \%$ of the working day, as there were not enough exposed subjects. When we used sitting $75 \%$ of the working day as a limit, no associations with the incidence of neck or shoulder pain were found for long term exposure, in concordance with results from the study already mentioned. However, changing from a more mobile job to sitting for more than $75 \%$ of the working day was significantly associated with neck or shoulder pain in our study.

Associations between perceived high workload and musculoskeletal pain have been found for the low back ${ }^{18}$ and neck or shoulder disorders, ${ }^{7}$ but only among women. In the present study, associations among women with short and long term perceived high workload were found. When the workload increases it may take some time to adapt and use coping strategies. The risk of seeking care for neck or shoulder pain is nearly double among those who perceived an increase in workload compared with those with a long term high level of workload. High perceived workload may be a measure of the total workload relative to a person's capacity. This is shown in the lowering of the risk estimates for this factor in the multivariate models. The most likely explanation for the lowering of the RR for perceived high workload when the 224 subjects who only responded to the questionnaire, almost all of them controls, were added to the study group, is that this group was not a random sample, but composed of people who, because of their high workload did not consider that they had enough time to participate in the whole study.

In the psychosocial factors, long term exposure to hindrance at work, an increase of exposure to reduced opportunities to acquire or use new knowledge, or lack of opportunity to participate in planning of the work are in this study associated with seeking care because of neck or shoulder pain. It has been found that psychological stress may result in muscle contractions. ${ }^{43}$ Pain provoked by psychosocial stress factors may, however, not be mediated through increased muscle activity. ${ }^{44}$

The labour market has undergone marked changes during recent years. The experience of being affected by changes without being able to influence anything, or seeing how skills that used to be of importance lose their relevance, seems to be associated with seeking care because of neck or shoulder pain. In the present study more people reported improved than worsened working conditions during the preceding 5 year period. It seems that for the few people for whom work has become more demanding, the risk of developing neck and shoulder pain has also increased.

\section{Interactive effects}

Musculoskeletal diseases have been found to have multifactorial causes. ${ }^{46745}$ In this study the long term effect of hindrance at work was increased by a concomitant presence of high perceived workload among women. Doing most of the daily household duties was by itself only faintly associated with neck or shoulder pain. However, interaction between this factor and physically strenuous conditions related to work was 
found, and points towards an overall overload. Among women, this interaction has been found in another study about psychosomatic strain. ${ }^{46}$ No interactive effects were identified among men. In other studies interactive effects on neck or shoulder disorders have been found among men, but not as often as among women. ${ }^{6}$ The results from the present study support the theory that neck or shoulder pain among women is associated with a combination of physical and psychosocial factors, both at work and in the family setting.

\section{Methodological considerations}

The study population comprised all people living and working in a geographically defined area. All caregivers in the same area, including caregivers outside the public health care and insurance system, sent their cases to the study. Therefore the chance of getting hold of all incident cases during the study period was fairly good. Before the study started estimations and power calculations were done. It was estimated that we should get around 700 cases of neck or shoulder pain. The number of cases turned out to be much smaller than expected partly due to the economic crises in Sweden during the first part of the 1990s. The crises resulted in a reduction of the number of people on the sick list and probably also of the number of people seeking care for musculoskeletal pain. The only way we could have got more subjects was to prolong the study period, but this was not financially possible. With the actual number of cases and controls in the study, the power of detecting RRs of 2 as significant for exposures with a prevalence of $25 \%$, was for women $98 \%$ and for men $80 \%$. For women the power was over $80 \%$ for exposures down to $10 \%$ prevalence.

Incidence of musculoskeletal pain is, however, ambiguous, as this type of pain tends to vary over time. ${ }^{47}$ The outcome, seeking care, is certainly not tantamount to neck or shoulder pain, but has the advantage of fixed criteria and excludes mild disorders that are not so serious, either for the affected person or for society. About $26 \%$ of the referents answered yes to questions about ongoing aches or pains in the questionnaire. Whether or not care is sought depends on many different factors, and whether or not it is possible to work despite neck or shoulder pain varies from job to job. Other factors are whether the patient can afford care, whether it is easy to get in contact with caregivers, and whether it is common in the patient's social group to seek care for musculoskeletal pain. People with chronic or recurrent neck or shoulder pain-that is, people who had previously sought care for neck or shoulder pain within the 6 month period before the current visits to caregivers, were excluded from the study. Others with chronic or recurrent pain might have sought care on earlier occasions, but found that they got little or no help. Therefore they chose not to seek care this time despite the pain. Another reason for not seeking out caregivers may be that the patients had previously been given advice and training instructions, and therefore this time tried to manage on their own. This might be one of the explanations for the low relative risks of seeking care which were found for long term exposure. The additional analyses of subjects who had not experienced neck or shoulder pain before ( $>7$ consecutive days), and were therefore presumed to reflect relatively recently developed cases and healthy referents, strengthen the findings in this study, that for most risk factors the induction period before the patient is driven to seek care for neck or shoulder pain is relatively short.

It is likely that caregivers forgot to report some cases, but such forgetfulness would hardly be selective towards people with special exposure patterns. The reliability of self administered retrospective questionnaires about physical conditions at work, has been found to be good, even up to 20 years back in time, with almost identical questions to those in this study. ${ }^{48}$ In studies of the validity of questionnaire based physical

\section{Main messages}

- Associations were found between several exposures in the work environment and seeking care for neck or shoulder pain.

- In women interactive effects were found between physical and psychosocial factors, both at work and in the family.

- The higher RRs for short term compared with long term exposure might indicate that willingness to seek care is highest when the neck or shoulder pain is a new experience.

exposure information, only minor differences about recall of exposure could be found between subjects with and without low back disorders. ${ }^{49}{ }^{50}$ Six year reproducibility results were found to be similar to previously reported test-retest results for physical exposure, ${ }^{49}$ indicating that the magnitude of recall error did not differ substantially within a 6 year period. In another study, questions about current physical working conditions in 1969 were repeated in 1993 about the 1969 conditions. ${ }^{51}$ Present neck or shoulder disorders influenced the recall of earlier working conditions only slightly and not systematically. When validating physical questionnaire data versus interview data in the present MUSIC study no differential misclassification was found. ${ }^{20}$ The interview based physical data have also been compared with observations and technical measurements, and the reliability between methods was found to be high. ${ }^{52}$ There is thus no reason to think that the results in the present study are seriously misclassified by outcome or exposure.

In the present study the questions about physical exposure were asked for both exposure over the past year and 5 years previously. It may be possible that a subject was exposed at both these times but not during the intervening years. It may also be possible that a subject was exposed during the intervening period but not at the chosen times. We have no control over these conditions, but as there is no reason to think that cases and referents should differ for these conditions, this will probably only cause a diluting effect on our results.

\section{CONCLUSIONS}

Associations between several exposures in the work environment and seeking care for neck or shoulder pain were found. The high RRs for short term exposure indicate that for many factors the induction periods for neck or shoulder pain may be short. Among women, an increased amount of VDT work, work in a seated position, work above shoulder level, and reduced opportunities to acquire new knowledge, and among men, an increased amount of sitting at work were associated with neck or shoulder pain. However, for repetitive work with the hands, hindrance at work, and local vibrations, longer induction periods seem to be required. Interactive effects between physical and psychosocial factors, both at work and in the family, were found, but only among women. The higher RRs for short term exposure compared with those for long term exposure might reflect that willingness to seek care is highest when the neck or shoulder pain is a new experience.

\section{ACKNOWLEDGEMENT}

This study was supported by grants from the Swedish Council for Work Life Research and the Stockholm County Council. We are very grateful to all caregivers in Norrtälje, and the administrators in the Norrtälje district of the Country Council.

\section{Authors' affiliations}

K Fredriksson, Å Kilbom, E Wigaeus Hielm, Programme for Ergonomics, National Institute for Working Life, Stockholm, Sweden K Fredriksson, Department of Physical Therapy, Karolinska Institutet, Huddinge, Sweden 
G Ahlberg, C Wiktorin, Department of Occupational Health, Stockholm County Council, Stockholm, Sweden

L Alfredsson, Division of Cardiovascular Epidemiology, Institute of

Environmental Medicine, Karolinska Institutet, Stockholm, Sweden

M Josephson, E Vingård, Department of Clinical Neuroscience,

Karolinska Institutet, Stockholm, Sweden

\section{REFERENCES}

1 Sommerich CM McGlothlin JD Marras WS Occupational risk factors associated with soft tissue disorders of the shoulder: a review of recent investigations in the literature. Ergonomics 1993;36:697-717.

2 Ohlsson K, Attewell RG, Pålsson BA, et al. Neck and upper limb disorders in females with repetitive industrial work. Am J Ind Med 1995;27:731-47.

3 Kilbom Å. Repetitive work of the upper extremity II. The scientific basis (knowledge base) for the guide. Int $J$ Ind Ergon 1994;14:59-86.

4 Linton SJ. Risk factors for neck and back pain in a working population in Sweden. Work and Stress 1990;4:41-9.

5 Winkel J, Westgaard R. Occupational and individual risk factors for shoulder-neck complaints: Part II. The scientific basis (literature review) for the guide. International Journal of Industrial Ergonomics 1992;10:85-104.

6 Fredriksson K, Alfredsson L, Köster M, et al. Risk factors for neck and upper limb disorders: results from a 24 years of follow up. Occup Environ Med 1999;56:59-66.

7 Fredriksson K, Alfredsson L, Bildt C, et al. Risk factors for neck and shoulder disorders: a nested case-control study covering a 24 year period. Am J Ind Med 2000;38:516-28.

8 Ekberg K, Biörkqvist B, Malm P, et al. Cross-sectional study of risk factors for symptoms in the neck and shoulder area. Ergonomics 1995;38:971-80.

9 Chiang $\mathrm{H}, \mathrm{Ko}$ Y, Chen S, et al. Prevalence of shoulder and upper-limb disorders among workers in the fish-processing industry. Scand J Work Environ Health 1993;19:126-31.

10 Punnett L, Fine L, Keyserling WM, et al. Shoulder disorders and postural stress in automobile assembly work. Scand J Work Environ Health 2000;26:283-91.

11 Kilbom Å, Persson J, Jonsson BG. Disorders of the cervicobrachial region among female workers in the electronics industry. International Journal of Industrial Ergonomics 1986;1:37-47.

12 Ariens GA, van Mechelen W, Bongers PM, et al. Physical risk factors for neck pain. Scand J Work Environ Health 2000;26:7-19.

13 Bongers $\mathbf{P}$, de Winter R, Kompier $M$, et al. Psychosocial factors at work and musculoskeletal diseases. Scand J Work Environ Health 1993;21:3-14.

14 Linton SJ. A review of psychological risk factors in back and neck pain Spine 2000;25: 1148-56

15 Holmström EB, Lindell J, Moritz U. Low back and neck/shoulder pain in construction workers: occupational workload and psychosocial risk factors. Spine 1992;17:672-7.

16 Barnekow-Bergkvist M, Hedberg GE, Janlert U, et al. Determinants of self-reported neck-shoulder and low back symptoms in a genera population. Spine 1998:23:235-43.

17 Rantanen J. Research challenges arising from changes in worklife. Scand J Work Environ Health 1999;25:473-83

18 Josephson M, Vingård E, MUSIC-Norrtälje study group. Workplace factors and care seeking for low back pain among female nursing personnel. Scand J Work Environ Health 1998;24:465-72.

19 Vingård $\mathbf{E}$, Alfredsson L, Hagberg $M$, et al. To what extent do current and past physical and psychosocial occupational factors explain care-seeking for low back pain in a working population? Results from the musculoskeletal intervention center Norrtälje study. Spine 2000;25:493-500

20 Wiktorin C, Vingard E, Mortimer M, et al. Interview versus questionnaire for assessing physical loads in the population-based MUSIC-Norrtalje study. Am J Ind Med 1999;35:44 1-55.

21 Mortimer M, Pernold G, Vingård E, et al. Inter-rater reliability of a physical examination of the low-back. 25th International Congress on Occupational Health. Stockholm: ICOH, 1996.

22 Waldenstrom $M$, Josephson $M$, Persson $C$, et al. Interview reliability for assessing mental work demands. J Occup Health Psychol 1998;3:209-16.

23 Borg G. Perceived exertion as an indicator of somatic stress. Scand J Rehabil Med 1970;2:92-8

24 Bergqvist U, Wolgast $E$, Nilsson $B$, et al. The influence of VDT work on musculoskeletal disorders. Ergonomics 1995;38:754-62.
25 Greiner B, Leitner K. Assessment of job stress: The RHIA instrument. Recent developments in job analysis. London: Taylor and Francis, 1989. 26 Bradburg NM, Rips L, Shevell SK. Answering autobiographical questions: the impact of memory and inference on surveys. Science 1987:236:157-61.

27 SAS. SAS/STAT user's guide, Cary, NC: SAS Institute, 1989

28 Miettinen OS. Estimability and estimation in case-referent studies. Am J Epidemiol 1976;103:226-35.

29 Rothman KJ. Modern epidemiology. Boston: Little Brown, 1986.

30 Stenlund B, Goldie I, Hagberg M, et al. Radiographic osteoarthrosis in the acromioclavicular joint resulting from manual work or exposure to vibration. Br J Ind Med 1992;49:588-93.

31 Vingård $\mathbf{E}$, Alfredsson L, Fellenius $\mathrm{E}$, et al. Disability pensions due to musculoskeletal disorders among men in heavy occupations. Scand J Soc Med 1992:20:31-6.

32 Andersen JH, Gaardboe O. Musculoskeletal disorders of the neck and upper limb among sewing machine operators: a clinical investigation. Am J Ind Med 1993;24:689-700.

33 Hägg G, Suurkula J, Kilbom Å. Predictors for work-related shoulder/neck disorders. A longitudinal study of female assembly workers. Arbete och Hälsa. Stockholm: The Swedish National Institute of Occupational Health, 1990: 10. (In Swedish, English summary.)

34 Kamwendo K, Linton S Moritz U. Neck and shoulder disorders in medical secretaries. Scand J Rehabil Med 1991;23:127-33.

35 Rossignol A Morse E, Summers V, et al. Video display terminal use and reported health symptoms among Massachusetts clerical workers. J Occup Med 1987;29:1 12-18.

36 Bielle A, Hagberg M, Michaelson G. Occupational and individual factors in acute shoulder-neck disorders among industrial workers. $\mathrm{Br} J$ Ind Med 1981:38:356-63.

37 Johansson H, Sojka P. Pathophysiological mechanisms involved in genesis and spread of muscular tension in occupational muscle pain and in chronic musculoskeletal pain syndromes: a hypothesis. Med Hypotheses 1991;35:196-203.

38 Hägg G. Static work load and occupational myalgia: a new explanation model. In: Anderson P, Hobart D, Danoff J, eds. Electromyographical kinesiology. Amsterdam: Elsevier, 1991:141-4.

39 Veiersted KB, Westgaard R, Andersen P. Electromyographic evaluation of muscular work patterns as a predictor of trapezius myalgia. Scand J Work Environ Health 1993:19:284-90.

40 Punnett L. Adjusting for the healthy worker selection effect in cross-sectional studies. Int J Epidemiol 1996;25:1068-76.

41 Oxenburg MS, Rowe SA, Douglas DB. Repetitive strain injury in keyboard operators, successful management over a 2-year period. $J$ Occupational Health and Safety, Australia and New Zealand $1985 ; 1: 106-12$

42 Ariens GA, van Mechelen W, Bongers PM, et al. Work-related neck flexion, neck rotation and sitting in relation to neck pain: results of a prospective cohort study. Singapore: 26th International Congress on Occupational Health, 2000

43 Lundberg U, Kadefors R, Melin B, et al. Psychophysiological stress and EMG activity of the trapezius muscle. International Journal of Behavioral Medicine 1994;1:354-70.

44 Vasselien OJ, Westgaard R. Can stress-related shoulder and neck pain develop independently of muscle activity? Pain 1996:64:221-30.

45 Bammer G. Review of current knowledge of musculoskeletal problems. Montreal: Work with display units 89, 1989

46 Hall EM. Gender, work control, and stress: A theoretical discussion and an empirical test. Int J Health Serv 1989;19:725-45.

47 Josephson $M$, Lagerström M, Hagberg $M$, et al. Musculoskeletal symptoms and job strain among nursing personnel: a study over a three-year period. Occup Environ Med 1997;54:681-5.

48 Torgén $M$, Alfredsson $L$, Köster $M$, et al. Reproducibility of a questionnaire for assessment of present and past physical activities. Int Arch Occup Environ Health 1997:70:107-18.

49 Torgén $M$, Alfredsson L, Winkel J, et al. Evaluation of questionnaire-based information on previous physical workloads. Scand J Work Environ Health 1999;25:246-54.

50 Pope DP, Silman AV, Cherry NM, et al. Validity of self-completed questionnaire measuring the physical demands of work. Scand J Work Environ Health 1998;24:376-85

51 Köster M, Alfredsson L, Michelsen $\mathrm{H}$, et al. Retrospective versus original information on physical and psychosocial exposure at work. Scand J Work Environ Health 1999:25:410-4.

52 Mortimer M, Wigaeus Hjelm E, Wiktorin C, et al. Validity of self-reported duration of work postures obtained by interview. Appl Ergon 1999;30:477-86. 